\title{
Long-Term Effects of Repetitive Transcranial Magnetic Stimulation on Markers for Neuroplasticity: Differential Outcomes in Anesthetized and Awake Animals
}

\author{
Roman Gersner, ${ }^{1}$ Elena Kravetz, ${ }^{1}$ Jodie Feil, ${ }^{1,2}$ Gaby Pell, ${ }^{1}$ and Abraham Zangen ${ }^{1}$ \\ ${ }^{1}$ Department of Neurobiology, Weizmann Institute of Science, Rehovot 76100, Israel, and ${ }^{2}$ Monash Alfred Psychiatry Research Centre, School of Psychology \\ and Psychiatry, Monash University, Melbourne, Victoria 3004, Australia
}

\begin{abstract}
Long-term effects of repetitive transcranial magnetic stimulation (rTMS) have been associated with neuroplasticity, but most physiological studies have evaluated only the immediate effects of the stimulation on neurochemical markers. Furthermore, although it is known that baseline excitability state plays a major role in rTMS outcomes, the role of spontaneous neural activity in metaplasticity has not been investigated. The first aim of this study was to evaluate and compare the long-term effects of high- and low-frequency rTMS on the markers of neuroplasticity such as BDNF and GluR1 subunit of AMPA receptor. The second aim was to assess whether these effects depend on spontaneous neural activity, by comparing the neurochemical alterations induced by rTMS in anesthetized and awake rats. Ten daily sessions of high- or low-frequency rTMS were applied over the rat brain, and $3 \mathrm{~d}$ later, levels of BDNF, GluR1, and phosphorylated GluR1 were assessed in the hippocampus, prelimbic cortex, and striatum. We found that high-frequency stimulation induced a profound effect on neuroplasticity markers; increasing them in awake animals while decreasing them in anesthetized animals. In contrast, low-frequency stimulation did not induce significant long-term effects on these markers in either state. This study highlights the importance of spontaneous neural activity during rTMS and demonstrates that high-frequency rTMS can induce long-lasting effects on BDNF and GluR1 which may underlie the clinical benefits of this treatment in neuroplasticity-related disorders.
\end{abstract}

\section{Introduction}

In recent years, brain stimulation has received widespread attention as a potential alternative therapy for psychiatric and neurologic disorders. Repetitive transcranial magnetic stimulation (rTMS) is a nonsurgical method for brain stimulation with minimal side effects (Rossi et al., 2009). Changes in TMS frequency and stimulation patterns can result in varying long-term effects. High-frequency stimulation $(>3 \mathrm{~Hz})$ generally results in facilitation - an effect that shares similarities with long-term potentiation (LTP), while low-frequency rTMS $(\leq 1 \mathrm{~Hz})$ induces reduction of synaptic efficiency-an effect that shares similarities with long-term depression (LTD) (Huang et al., 2005; Houdayer et al., 2008). Nevertheless, little is known about the long-lasting neurochemical and physiological effects of rTMS, because most neurobiological studies have focused on the immediate effects induced after the stimulation.

Glutamate transmission plays a fundamental role in LTP and LTD, which depend on the influx of calcium ions in the postsynaptic cell (Bear and Malenka, 1994). Increased calcium activates different calcium-sensitive signaling pathways and induces longterm changes in both the presynaptic and postsynaptic neuron,

Received Dec. 26, 2010; revised March 30, 2011; accepted April 8, 2011.

Author contributions: R.G. and A.Z. designed research; R.G., E.K., J.F., and A.Z. performed research;R.G., E.K., G.P., and A.Z. analyzed data; R.G., J.F., G.P., and A.Z. wrote the paper.

Correspondence should be addressed to Dr. Abraham Zangen, Department of Neurobiology, Weizmann Institute of Science, Rehovot 76100, Israel. E-mail: a.zangen@weizmann.ac.il.

DOI:10.1523/JNEUROSCI.6751-10.2011

Copyright $\odot 2011$ the authors $\quad 0270-6474 / 11 / 317521-06 \$ 15.00 / 0$ which leads to increased synaptic strength (Malenka and Bear, 2004). Two significant consequences of this process are the insertion of additional AMPA receptors (AMPARs) in the postsynaptic neuron and the direct phosphorylation of existing AMPARs (Thickbroom, 2007), especially at the site of their GluR1 subunit (giving rise to pGluR1). This in turn leads to increased receptor permeability to calcium (Roche et al., 1996). The role of AMPARs in the mechanism of rTMS has been suggested (Hoogendam et al., 2010) but has yet to be demonstrated.

According to the synaptic consolidation hypothesis (Bramham and Messaoudi, 2005), neurotrophins such as brain-derived neurotrophic factor (BDNF) regulate synaptic strength and mediate plasticity. Moreover, different forms of BDNF are implicated in the mechanisms of LTP (Barco et al., 2005) and LTD (Martinowich et al., 2007). An effect of rTMS on BDNF levels has been suggested; however, only immediate or short-lasting alterations were studied with wide-ranging variability in the results, especially for low-frequency rTMS (see Discussion). These discrepancies may result from various factors such as different stimulation parameters (Hoogendam et al., 2010; Pell et al., 2011), or variations in the neural activity of the stimulated region. Indeed, the brain state at the time of stimulation influences the physiological and behavioral outcomes as manifested, for example, by the homeostatic mechanisms of priming (Amiaz et al., 2009; Siebner et al., 2009; Stehberg et al., 2009). The influence of altered states of spontaneous neural activity on this brain state dependence, however, has not been investigated. The difference between awake and anesthetized states serves as an ideal model to 


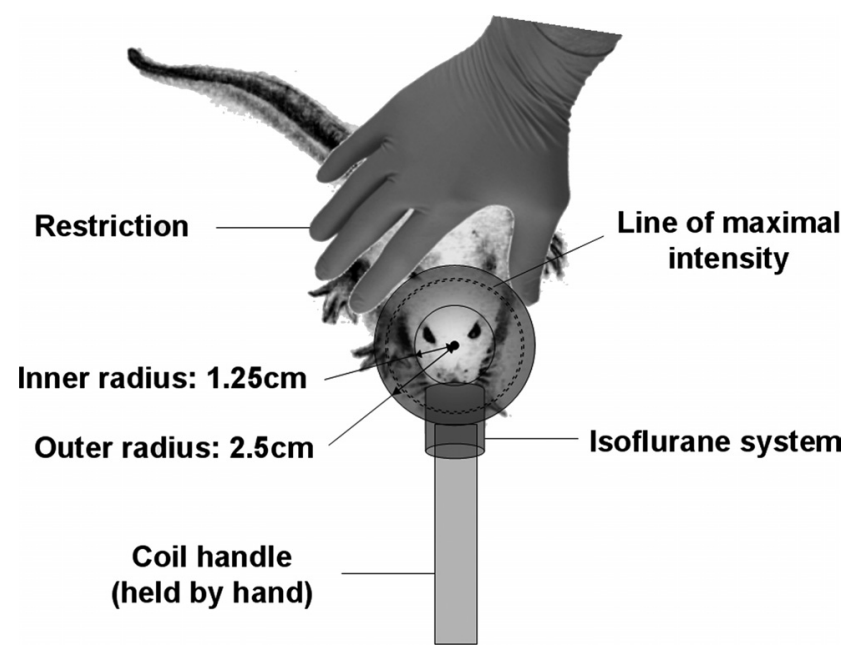

Figure 1. Diagram of rat stimulation. The circular coil was positioned over the rat's head so that the coil center was placed over the middle of the interocular line with the handle pointing forward. The awake groups were restrained by hand, whereas the anesthesia in the other groups was performed using the isoflurane system.

investigate this influence since stereotypical alterations in spontaneous neural activity are characteristic of the anesthesia (Galloway et al., 2010).

While previous studies have evaluated short-term neurochemical and physiological effects of rTMS, the characterization of long-lasting effects is crucial for enhancing the clinical utility of the method. Thus, in the present study we aimed to study the lasting effect of low- and high-frequency TMS on neurochemical markers of brain plasticity, such as BDNF and AMPAR. To test whether the long-term effects of stimulation on these markers depend on altered states of spontaneous neural activity, we compared the effects of rTMS on awake and anesthetized animals.

\section{Materials and Methods}

Animals. Male Sprague Dawley rats (60 d old at experiment initiation) were maintained under a $12 \mathrm{~h}$ light/dark cycle with food and water ad libitum. Rats were singly housed in Perspex cages $(18 \times 26 \times 40 \mathrm{~cm})$. All animal experiments were conducted according to the Institutional Animal Use and Care Committee guidelines.

TMS. Repetitive TMS was administered daily for $10 \mathrm{~d}$ during a 2 week period, and brains were removed $3 \mathrm{~d}$ after the last stimulation to measure lasting effects on markers for neuroplasticity. Stimulation was applied using a small circular coil (inner diameter, $2.5 \mathrm{~cm}$; outer diameter, $5 \mathrm{~cm}$; Brainsway Inc.) at $60 \%$ of the maximal output of a Rapid ${ }^{2}$ stimulator (Magstim). The coil was placed over the rat's head as described in Figure 1 to allow maximal stimulation over frontal cortex. The stimulation was performed on 36 awake and 32 anesthetized (with isoflurane vaporized in pure oxygen; $4 \%$ for induction, $2 \%$ during treatment) rats. All rats were randomly assigned to one of three groups: low-frequency, highfrequency, and sham stimulation ( $n=8-12$ per group). The intensity of stimulation represented $120 \%$ of the average resting motor threshold (as determined by visual inspection of bilateral forelimb movement in a preliminary experiment) in anesthetized animals and was kept constant in all rats of all groups. A total of 900 daily magnetic stimulation pulses were applied to both high- and low-frequency-stimulated groups. Lowfrequency daily stimulation was applied continuously at $1 \mathrm{~Hz}$ for $900 \mathrm{~s}$. High-frequency daily stimulation consisted of 9 trains of 100 pulses delivered at $20 \mathrm{~Hz}$ ( $5 \mathrm{~s}$ each train) with an intertrain interval of $55 \mathrm{~s}$ to allow effective cooling of the coil, and keeping time frame similar to that in the low-frequency stimulation groups. Restriction of the awake rats during stimulation was performed by hand force (Fig. 1). To control for differences in acute stress levels in the awake animals (resulting from the stimulation and need to restrain the rats), the sham awake group was
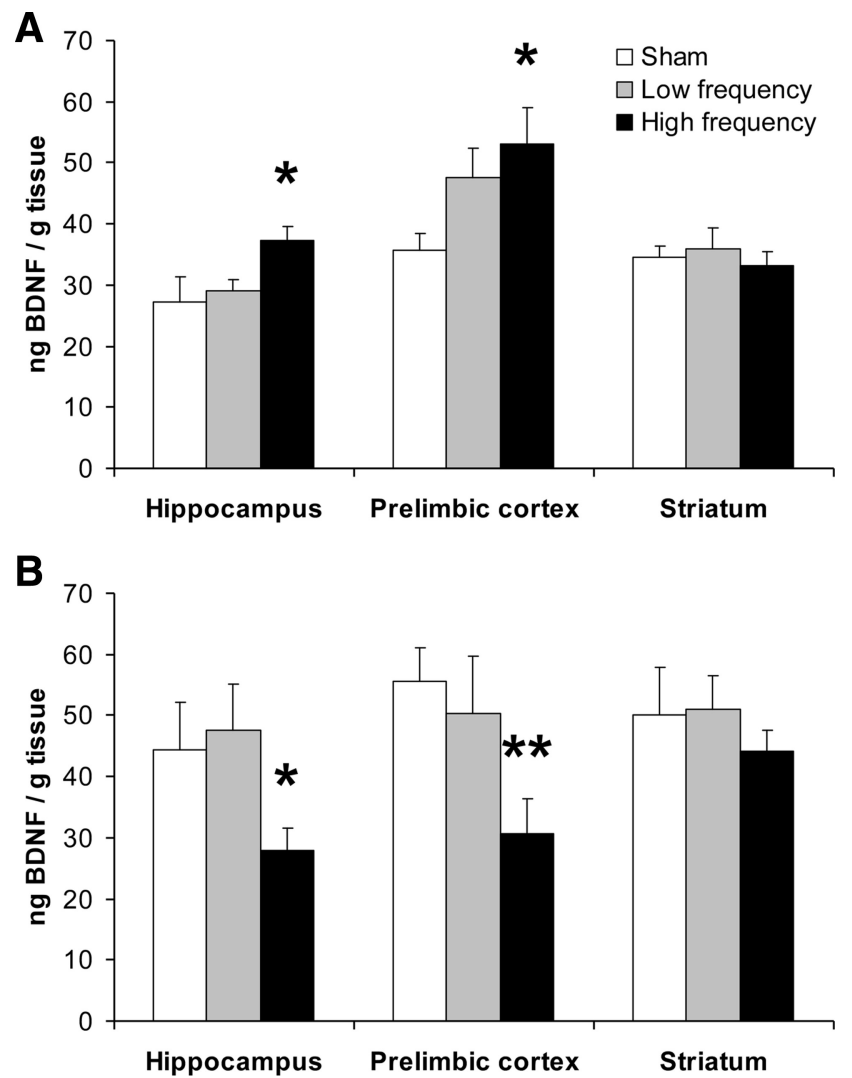

Figure 2. Effects of rTMS on BDNF expression. Data are presented as mean \pm SEM of BDNF levels in the hippocampus, prelimbic cortex, and striatum of animals previously treated with 10 daily rTMS or sham rTMS sessions while awake $(\boldsymbol{A})$ or anesthetized $(\boldsymbol{B}){ }^{*} p<0.05,{ }^{* *} p<0.01$, compared with the sham control group.

divided between low- and high-frequency stimulation. All sham animals were handled in a manner similar to that of the treatment groups (awake or anesthetized), but the magnetic stimulation was shielded by mu metal and did not induce motor activation.

Histology and tissue punches. All tissue preparations and histology were performed blind to experimental conditions. To analyze lasting effects of the treatment on regional levels of BDNF and GluR1, brains were removed $3 \mathrm{~d}$ after the last stimulation, frozen in isopropanol, and stored at $-80^{\circ} \mathrm{C}$. Coronal sections were sliced using a cryostat at $-20^{\circ} \mathrm{C}$ to reach the appropriate region, and then tissue punches were extracted from the prelimbic cortex (from $+3.7 \mathrm{~mm}$ to $+2.2 \mathrm{~mm}$ relative to bregma), striatum (from +2.2 to $+0.7 \mathrm{~mm}$ ), and dorsal hippocampus (from -2.3 to $-4.3 \mathrm{~mm}$ ) using a manual cutter, as described previously (Gersner et al., 2010; Taliaz et al., 2010). Extracted proteins were assayed with ELISA (for BDNF analysis) and Western blot (for GluR1 and pGluR1 analysis).

ELISA. Sandwich ELISA was performed using monoclonal mouse anti-human BDNF capture and detection antibodies (R\&D Systems) at $2.0 \mu \mathrm{g} / \mathrm{ml}$ in PBS, as described previously (Toth et al., 2008). The color was developed using an HRP system and the plates were read at $450 \mathrm{~nm}$.

Western blot. Western blot was performed using polyclonal antibodies against GluR1 (Millipore Bioscience Research Reagents), pGluR1 (antiSer845, Millipore Bioscience Research Reagents), and $\beta$-actin (Abcam). Probes were developed using an Odyssey Infrared Imaging System with goat-anti-rabbit IRDye $800 \mathrm{CW}$ secondary antibody (LI-COR Biosciences). To analyze the data, results were normalized to actin levels and presented as percentage of sham.

Statistical analysis. Results are presented as means \pm SEM. Data from each experiment were analyzed using one-way ANOVA, followed by Fisher's least significant difference post hoc test comparing both treatment groups to the sham group. The correlations between levels of BDNF and GluR1 or pGluR1 were assessed by linear regression analysis. Since no differences in levels of BDNF, GluR1, and 

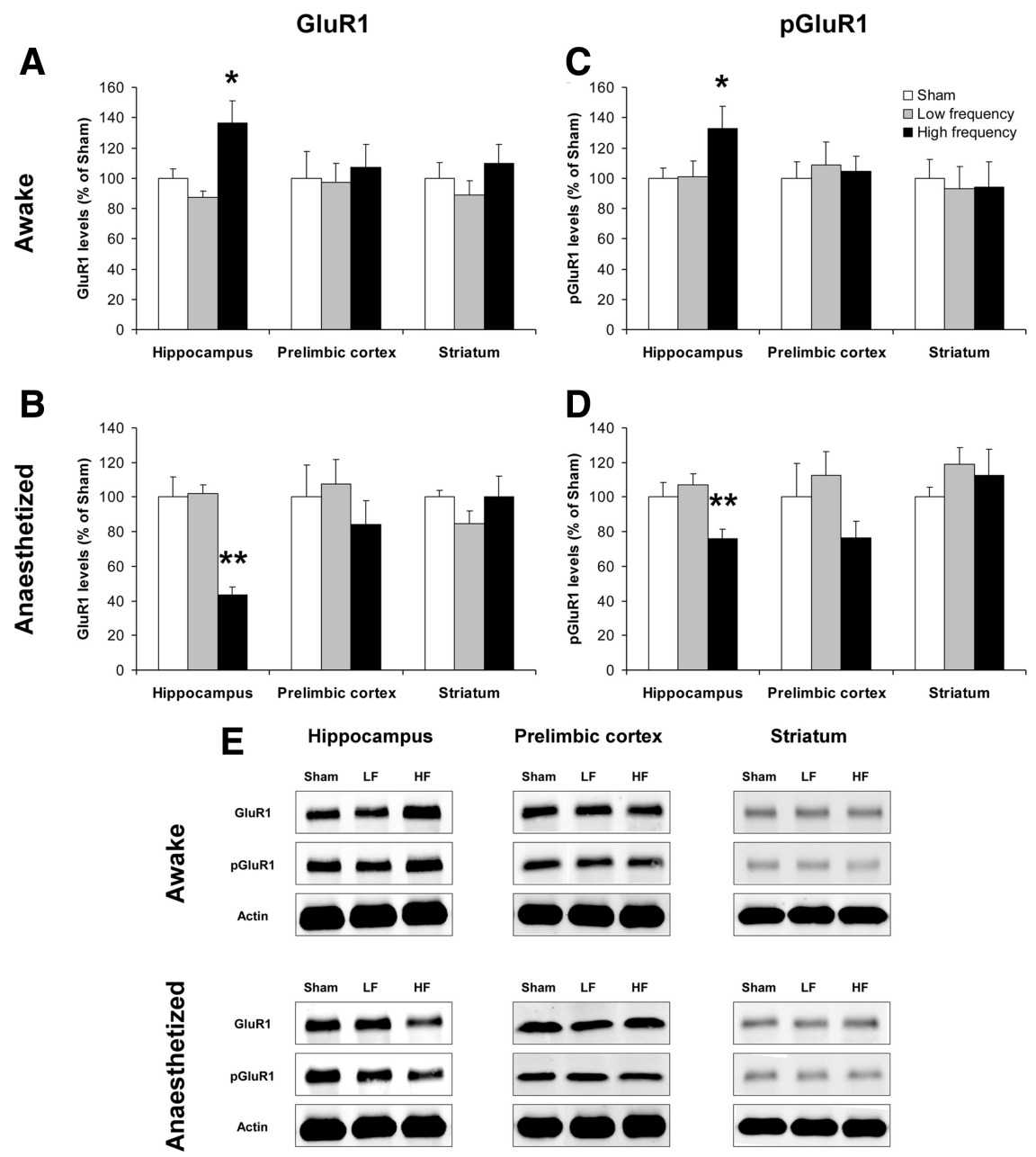

Figure 3. Effects of rTMS on GluR1 and pGluR1 levels. $\boldsymbol{A}-\boldsymbol{D}$, Data are presented as mean \pm SEM of GluR1 $(\boldsymbol{A}, \boldsymbol{B})$ and pGluR1 ( $\boldsymbol{C}$, $D$ ) relative levels in the hippocampus, prelimbic cortex, and striatum of animals previously treated with 10 daily rTMS or sham rTMS sessions while awake $(\boldsymbol{A}, \boldsymbol{C})$ or anesthetized $(\boldsymbol{B}, \boldsymbol{D})$. $\boldsymbol{E}$, Representative Western blots of GluR1, pGluR1, and corresponding actin in the measured areas of all treatment groups are shown. ${ }^{*} p<0.05,{ }^{* *} p<0.01$, compared with the sham control group. LF, Low frequency; HF, high frequency.

pGluR1 were observed between the awake groups receiving sham stimulation at low frequency or high frequency [e.g., hippocampal BDNF, $29.8 \pm 7.9$ (low-frequency sham) vs $25.2 \pm 3.4$ (highfrequency sham); prelimbic cortex BDNF, $32.5 \pm 4.6$ vs $39.1 \pm 2.5$; striatum, $34.3 \pm 2.7$ vs $34.7 \pm 2.9, p>0.2$ for all sham comparisons), both sham conditions in awake rats were combined into a single group. All statistical analysis was performed with StatView 5.0 software. A $p$ value of $<0.05$ was considered statistically significant.

\section{Results}

Effects of rTMS on BDNF levels in awake animals

Ten sessions of rTMS in awake animals induced significant alterations in BDNF levels in the hippocampus $\left(F_{(2,31)}=3.55, p=\right.$ 0.04; Fig. 2A). Post hoc analysis revealed that high-frequency stimulation caused a significant increase in hippocampal BDNF levels ( $p=0.019$ ), while the low-frequency rTMS had no effect relative to the sham controls.

A significant effect of rTMS in awake animals was also observed in the prelimbic cortex $\left(F_{(2,33)}=3.72, p=0.039\right)$. Post hoc analysis revealed that the high-frequency stimulation caused a significant elevation in BDNF levels $(p=0.01)$, while the lowfrequency induced a nonsignificant trend $(p=0.078)$ to increase prelimbic BDNF levels, relative to the sham controls.
No effects of rTMS on BDNF levels in the striatum were detected (Fig. 2A).

\section{Effects of rTMS on BDNF levels in anesthetized animals}

In anesthetized animals, a similar treatment with 10 rTMS sessions induced significant $\left(F_{(2,27)}=3.27, p=0.0497\right.$; Fig. $2 B)$, but opposite, effects on hippocampal BDNF levels. Post hoc analysis revealed that high-frequency stimulation in the anesthetized animals caused a significant decrease in hippocampal BDNF levels ( $p=0.025$ ), while low-frequency stimulation had no effect relative to the sham controls.

Similarly, in the prelimbic cortex of the anesthetized rTMS-treated animals, a significant $\left(F_{(2,27)}=3.56, p=0.042\right)$ reduction in BDNF levels was observed, and post hoc analysis revealed again that only high-frequency rTMS decreased BDNF levels $(p=0.022)$, while the lowfrequency rTMS had no effect relative to the sham controls.

In the striatum, no effects of rTMS on BDNF levels were detected (Fig. 2B).

\section{Effects of rTMS on GluR1 and pGluR1 levels in awake animals}

The 10 sessions of rTMS in awake animals affected both GluR1 and pGluR1 levels in the hippocampus (GluR1, $F_{(2,33)}=7.05$, $p=0.003$; pGluR1, $F_{(2,33)} \stackrel{=}{=} 2.9, p=$ $0.047)$, but not in the other tested regions. Post hoc analysis revealed that hippocampal GluR1 ( $p=0.01$; Fig. $3 A)$ and pGluR1 $(p=0.041$; Fig. $3 C$ ) levels were significantly increased following high-frequency but not low-frequency rTMS, relative to the sham controls.

\section{Effects of rTMS on GluR1 and pGluR1 levels in anesthetized animals}

Again, in anesthetized animals an opposite effect of rTMS on hippocampal GluR1 and pGluR1 levels was observed (GluR1, $F_{(2,27)}=25.65, p<0.0001$; pGluR1, $\left.F_{(2,27)}=7.33, p=0.003\right)$. Post hoc analysis revealed that the high-frequency rTMS significantly decreased hippocampal GluR1 ( $p<0.0001$; Fig. $3 B)$ and pGluR1 ( $p=0.014$; Fig. $3 D$ ) levels. No effects of the rTMS treatment on GluR1 and pGluR1 levels were observed in the prelimbic cortex or the striatum.

\section{Correlation between BDNF and GluR1 levels}

The general effects of rTMS on hippocampal BDNF and GluR1 or pGluR1 levels were quite similar and, therefore, the correlations between these neurochemical outcomes were assessed. In awake-treated animals (Fig. $4 A$ ) a significant correlation was found between BDNF and GluR1 $\left(R^{2}=0.314, p=0.0009\right)$ and between BDNF and pGluR1 $\left(R^{2}=0.149, p=0.029\right)$ levels. Similarly, a significant correlation was found in hippocampus of anesthetized-treated animals between BDNF and 

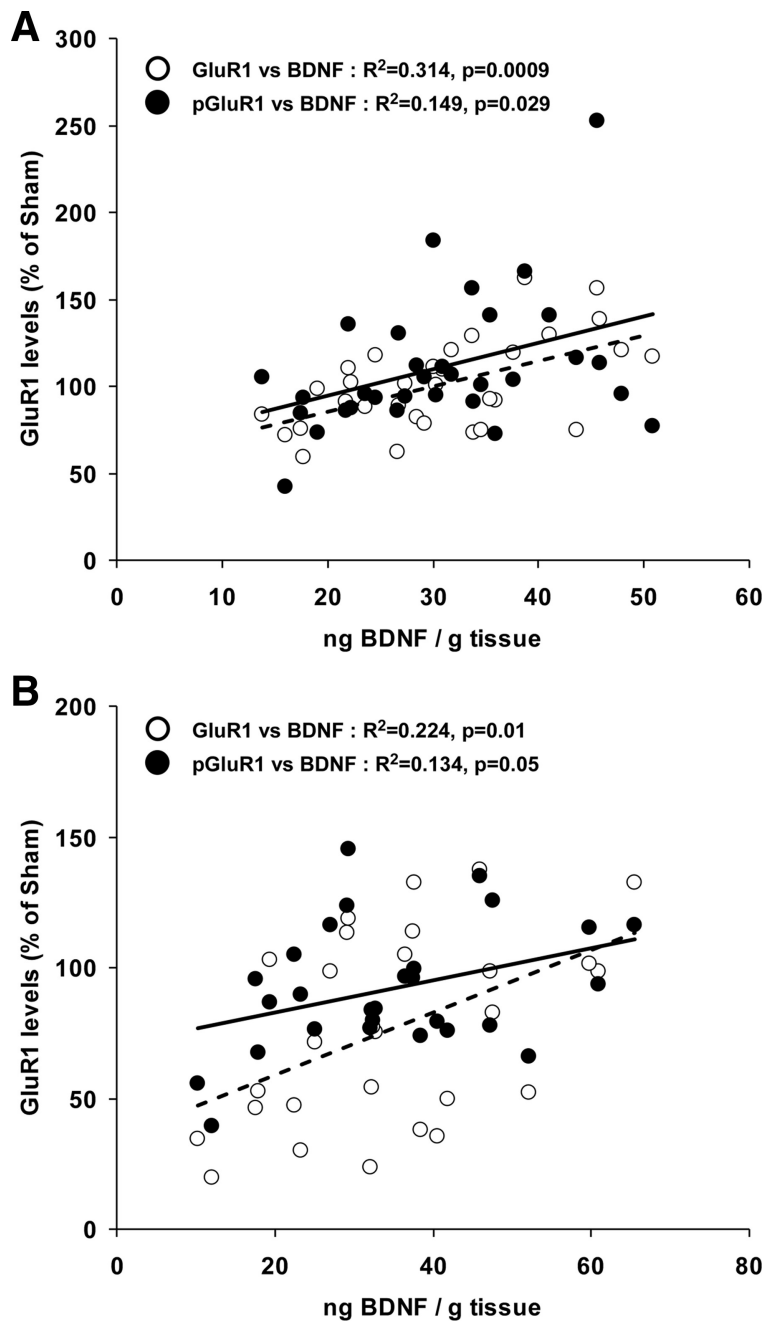

Figure 4. Correlations between BDNF and GluR1 or pGluR1 levels in the hippocampus. BDNF levels are plotted as a function of GluR1 (empty circles) or pGluR1 (black circles) relative levels in the hippocampus of animals previously treated with 10 daily rTMS or sham rTMS sessions while awake $(\boldsymbol{A})$ or anesthetized $(\boldsymbol{B})$. The corresponding linear fit is presented.

GluR1 $\left(R^{2}=0.224, p=0.01\right)$ and between BDNF and pGluR1 $\left(R^{2}=0.134, p=0.048\right)$ levels (Fig. $\left.4 B\right)$.

\section{Discussion}

It has been previously suggested that rTMS can induce alterations in neuroplasticity and thereby possesses therapeutic potentials (Hallett, 2000). However, evidence for long-lasting, rather than immediate, effects of several rTMS sessions on neuroplasticity is lacking. The present study identifies long-term alterations in neurochemical markers for neuroplasticity that are induced by 10 sessions of high-frequency (but not low-frequency) rTMS. Moreover, the present study indicates that the brain state (i.e., alterations in neural activity induced by anesthesia) during the stimulation has critical effects on the lasting neurochemical outcomes of rTMS treatment.

While a different brain stimulation technique, termed transcranial direct current stimulation (tDCS), was recently shown to promote BDNF-dependent synaptic plasticity (Fritsch et al., 2010), previous studies reported mixed results on effects of rTMS on BDNF levels. In animal models, both high-frequency (Müller et al., 2000) and low-frequency (Zhang et al., 2007) rTMS induced fast increases in BDNF levels. In a cell culture system, low-frequency $(0.6 \mathrm{~Hz})$ rTMS caused increases or decreases in BDNF transcription, depending on the specific cell lines (Henkel et al., 2008). In humans, BDNF was measured in the blood serum, which may not directly correlate with the brain levels, and therefore provides a less direct marker for changes in neuroplasticity. In healthy control subjects, low-frequency rTMS reduced serum BDNF levels in one study (Angelucci et al., 2004), but had no effect in another (Lang et al., 2008), while the effect of highfrequency rTMS has not been reported. The effect of rTMS on BDNF levels in depressed subjects has received considerably greater attention because of the strong links between BDNF and the pathophysiology of depression (Krishnan and Nestler, 2008; Taliaz et al., 2010). In depressed patients who responded to TMS treatment, both high-frequency (Yukimasa et al., 2006; Zanardini et al., 2006) and low-frequency (Zanardini et al., 2006) rTMS were reported to increase BDNF serum levels; however, a different study reported no change induced by high-frequency rTMS (Lang et al., 2006).

In the present study, unlike the significant lasting effects of high-frequency rTMS, the low-frequency rTMS did not induce lasting effects on BDNF, GluR1, or pGluR1 levels in any of the measured regions, in either animal state. It is important to mention that such stimulation differs not only in its frequency but also in the overall pattern (intermittent vs continuous; see Materials and Methods), and this may also play a role in the differential lasting outcomes on markers for neuroplasticity, especially given that different stimulation frequencies can result in differential activation of immediate early genes in the rat brain (AydinAbidin et al., 2008). While low-frequency electrical stimulation generally leads to LTD-like effects (Hoffman and Cavus, 2002; Houdayer et al., 2008), the induction of this form of plasticity modulation in vivo has always proved a greater challenge (Errington et al., 1995; Abraham et al., 1996). The responses to lowfrequency stimulation are generally more variable, and the aftereffect durations are shorter (Gangitano et al., 2002; Fitzgerald et al., 2006). Therefore, the absence of effect as measured $3 \mathrm{~d}$ after the last stimulation session may not be surprising and emphasizes the need to reevaluate the potential long-term clinical benefits of low-frequency rTMS.

Recent literature suggests that rTMS effects are strongly dependent on the brain state at the time of stimulation as determined by factors like history of synaptic activity and behavioral engagement, and manifested by the mechanisms of gating and priming (Siebner et al., 2009). Similar to a previous study reporting increased BDNF levels induced within $20 \mathrm{~h}$ after several rTMS sessions (Müller et al., 2000), we found that high-frequency rTMS in awake animals led to a long-term (3 d) increase in hippocampal BDNF levels. However, in our study, somewhat surprisingly, it was also shown that the exact same stimulation protocol in anesthetized animals leads to a significant decrease in BDNF levels. Our result therefore emphasizes that the brain state as reflected by baseline excitability and spontaneous neural activity may have a crucial effect on lasting rTMS outcomes. It is important to note that the reasons for the disparity between the two states in this study are likely multifaceted and may result from differences in stress levels, auditory arousal, and the effect of anesthetics. Indeed, volatile anesthesia (such as isoflurane) decreases excitatory and increases inhibitory transmission (Gomez and Guatimosim, 2003; Ouyang and Hemmings, 2005), affects neuroplasticity, and can alter BDNF expression (Lu et al., 2006; Head et al., 2009).

Two potential explanations can be put forward for the statedependent effects observed in this study. One explanation is 
based on TMS-induced membrane potential modulation. Neuronal response to TMS pulses implicates spatially and temporally differentiated areas of depolarization and hyperpolarization. Repeated induction of depolarization/hyperpolarization will influence long-term excitability not only via classical synaptic mechanisms (similar to LTP and LTD), but also by their direct influence on membrane potential (Chan et al., 2002; Pell et al., 2011). The inhibitory characteristics of hyperpolarization are considered crucial in homeostatic regulation to counterbalance the destabilizing influences of Hebbian synaptic plasticity (Dan and Poo, 2006). Periods of both reduced excitability (hyperpolarization) and increased excitability (depolarization) are apparent, with hyperpolarization dominating the long-term response. Similarly, the response to a train of repeated impulses is dominated by sustained reduced excitability, unless a prolonged period of high-frequency tetanic stimulation is used, when a transient increase in excitability can be observed (Applegate and Burke, 1989). Against this background, high-frequency rTMS is similarly capable of transitioning the system to a temporary "non-equilibrium" state of increased depolarization-induced excitability. In the presence of isoflurane anesthesia, the ability of high-frequency rTMS to depolarize is, however, weakened. The balance between the two excitability states that is normally weighted by the high-frequency stimulation toward increased excitability may be disturbed and, instead, tipped toward the equilibrium state represented by reduced excitability. This state is manifested by LTD-like effects, with inhibited excitatory synaptic transmission (Malenka and Bear, 2004) and decreased BDNF levels (Aicardi et al., 2004).

An alternative explanation is based on non-homeostatic metaplasticity. In recently published work (Nitsche et al., 2007), the application of tDCS before excitatory paired-associative stimulation (PAS) caused a non-homeostatic response. In this case, previous excitability-enhancing anodal tDCS ("gating") boosted the efficacy of excitatory PAS. In contrast, previous inhibitory cathodal tDCS ("anti-gating") did not just block PAS facilitation but instead gave rise to inhibition. This "synergism" can be described in terms of calcium levels, such that the "anti-gating intervention" induced by isoflurane may reduce the net calcium influx to a level below the threshold for inducing LTP-like plasticity via protein kinase activation, while still causing a small intracellular calcium increase that is sufficient to trigger specific protein phosphatases that lead to LTD-like plasticity (Bear and Malenka, 1994). This would change the sign of plasticity from LTP-like to LTD-like plasticity (Siebner, 2010), therefore causing a decrease in BDNF expression (Aicardi et al., 2004).

Current TMS coils for rodents cannot be used to localize stimulation to specific brain regions because of technical limitations of the coil size relative to the rat brain. In the present study, the coil was positioned to allow maximal field intensity over frontal cortex; however, all other brain areas could be affected as well. Therefore, differences observed in this study between rTMS effects on the hippocampus, prelimbic cortex, and striatum may merely reflect the differing neuroplastic nature of these regions. While we did not find any lasting effects of rTMS on striatal BDNF or GluR1 levels, the lasting effects of high-frequency rTMS on BDNF expression in the prelimbic cortex were similar to those found in the hippocampus. This may reflect the strong excitatory interconnection between the prelimbic cortex and the hippocampus (Vertes, 2006). Expectedly, the effect of TMS on GluR1 and pGluR1 levels not only mirrored changes in BDNF levels in the hippocampus but also positively correlated. This correlation is also supported by previous studies showing that positive AMPAR modulation increases BDNF expression and facilitates LTP induction (Jourdi et al., 2009). The GluR1 and pGluR1 levels in prelimbic cortex and striatum remained unaffected by rTMS in both states, indicating lower plasticity in these regions relative to the hippocampus. Interestingly, in these regions, contrary to the hippocampus, GluR1 and pGluR1 levels were not correlated with BDNF levels.

The main goal of the present study was to evaluate potential long-lasting outcomes of standard rTMS protocols; however, it is important to mention that using different stimulation intensities and/or patterns may have significant effects on the long-lasting outcomes (e.g., increasing the intensity may even reverse LTD-like effects to LTP-like effects). Therefore, effects such as stimulation intensity and number of sessions on long-lasting alterations in markers for neuroplasticity warrant future studies.

In summary, the present study shows lasting effects of highfrequency (but not low-frequency) rTMS on markers for neuroplasticity, and indicates that spontaneous neural activity during the stimulation can dramatically affect the neurochemical outcomes. The fact that alterations in these markers remained $3 \mathrm{~d}$ after the last rTMS session may have implications on the potential clinical use of rTMS. Moreover, the characterization of long-term effects on markers such as BDNF improves our understanding of mechanisms underlying the clinical benefits obtained with rTMS in the treatment of depression and other psychiatric or neurological disorders.

\section{References}

Abraham WC, Mason-Parker SE, Logan B (1996) Low-frequency stimulation does not readily cause long-term depression or depotentiation in the dentate gyrus of awake rats. Brain Res 722:217-221.

Aicardi G, Argilli E, Cappello S, Santi S, Riccio M, Thoenen H, Canossa M (2004) Induction of long-term potentiation and depression is reflected by corresponding changes in secretion of endogenous brain-derived neurotrophic factor. Proc Natl Acad Sci U S A 101:15788-15792.

Amiaz R, Levy D, Vainiger D, Grunhaus L, Zangen A (2009) Repeated highfrequency transcranial magnetic stimulation over the dorsolateral prefrontal cortex reduces cigarette craving and consumption. Addiction 104:653-660

Angelucci F, Oliviero A, Pilato F, Saturno E, Dileone M, Versace V, Musumeci G, Batocchi AP, Tonali PA, Di Lazzaro V (2004) Transcranial magnetic stimulation and BDNF plasma levels in amyotrophic lateral sclerosis. Neuroreport 15:717-720.

Applegate C, Burke D (1989) Changes in excitability of human cutaneous afferents following prolonged high-frequency stimulation. Brain 112:147-164.

Aydin-Abidin S, Trippe J, Funke K, Eysel UT, Benali A (2008) High- and low-frequency repetitive transcranial magnetic stimulation differentially activates c-Fos and zif268 protein expression in the rat brain. Exp Brain Res 188:249-261.

Barco A, Patterson SL, Alarcon JM, Gromova P, Mata-Roig M, Morozov A, Kandel ER (2005) Gene expression profiling of facilitated L-LTP in VP16-CREB mice reveals that BDNF is critical for the maintenance of LTP and its synaptic capture. Neuron 48:123-137.

Bear MF, Malenka RC (1994) Synaptic plasticity: LTP and LTD. Curr Opin Neurobiol 4:389-399.

Bramham CR, Messaoudi E (2005) BDNF function in adult synaptic plasticity: the synaptic consolidation hypothesis. Prog Neurobiol 76:99-125.

Chan JH, Lin CS, Pierrot-Deseilligny E, Burke D (2002) Excitability changes in human peripheral nerve axons in a paradigm mimicking paired-pulse transcranial magnetic stimulation. J Physiol 542:951-961.

Dan Y, Poo MM (2006) Spike timing-dependent plasticity: from synapse to perception. Physiol Rev 86:1033-1048.

Errington ML, Bliss TV, Richter-Levin G, Yenk K, Doyère V, Laroche S (1995) Stimulation at $1-5 \mathrm{~Hz}$ does not produce long-term depression or depotentiation in the hippocampus of the adult rat in vivo. J Neurophysiol 74:1793-1799.

Fitzgerald PB, Fountain S, Daskalakis ZJ (2006) A comprehensive review of 
the effects of rTMS on motor cortical excitability and inhibition. Clin Neurophysiol 117:2584-2596.

Fritsch B, Reis J, Martinowich K, Schambra HM, Ji Y, Cohen LG, Lu B (2010) Direct current stimulation promotes BDNF-dependent synaptic plasticity: potential implications for motor learning. Neuron 66:198-204.

Galloway GM, Nuwer MR, Lopez JR, Zamel KM (2010) Intraoperative neurophysiologic monitoring. Cambridge, UK: Cambridge UP.

Gangitano M, Valero-Cabré A, Tormos JM, Mottaghy FM, Romero JR, Pascual-Leone A (2002) Modulation of input-output curves by low and high frequency repetitive transcranial magnetic stimulation of the motor cortex. Clin Neurophysiol 113:1249-1257.

Gersner R, Toth E, Isserles M, Zangen A (2010) Site-specific antidepressant effects of repeated subconvulsive electrical stimulation: potential role of brain-derived neurotrophic factor. Biol Psychiatry 67:125-132.

Gomez RS, Guatimosim C (2003) Mechanism of action of volatile anesthetics: involvement of intracellular calcium signaling. Curr Drug Targets CNS Neurol Disord 2:123-129.

Hallett M (2000) Transcranial magnetic stimulation and the human brain. Nature 406:147-150.

Head BP, Patel HH, Niesman IR, Drummond JC, Roth DM, Patel PM (2009) Inhibition of p75 neurotrophin receptor attenuates isoflurane-mediated neuronal apoptosis in the neonatal central nervous system. Anesthesiology 110:813-825.

Henkel AW, Sperling W, Rotter A, Reulbach U, Reichardt C, Bönsch D, Maler JM, Kornhuber J, Wiltfang J (2008) Antidepressant drugs modulate growth factors in cultured cells. BMC Pharmacol 8:6.

Hoffman RE, Cavus I (2002) Slow transcranial magnetic stimulation, longterm depotentiation, and brain hyperexcitability disorders. Am J Psychiatry 159:1093-1102.

Hoogendam JM, Ramakers GM, Di Lazzaro V (2010) Physiology of repetitive transcranial magnetic stimulation of the human brain. Brain Stimul 3:95-118.

Houdayer E, Degardin A, Cassim F, Bocquillon P, Derambure P, Devanne H (2008) The effects of low- and high-frequency repetitive TMS on the input/output properties of the human corticospinal pathway. Exp Brain Res 187:207-217.

Huang YZ, Edwards MJ, Rounis E, Bhatia KP, Rothwell JC (2005) Theta burst stimulation of the human motor cortex. Neuron 45:201-206.

Jourdi H, Hsu YT, Zhou M, Qin Q, Bi X, Baudry M (2009) Positive AMPA receptor modulation rapidly stimulates $\mathrm{BDNF}$ release and increases dendritic mRNA translation. J Neurosci 29:8688-8697.

Krishnan V, Nestler EJ (2008) The molecular neurobiology of depression. Nature 455:894-902.

Lang UE, Bajbouj M, Gallinat J, Hellweg R (2006) Brain-derived neurotrophic factor serum concentrations in depressive patients during vagus nerve stimulation and repetitive transcranial magnetic stimulation. Psychopharmacology (Berl) 187:56-59.

Lang UE, Hellweg R, Gallinat J, Bajbouj M (2008) Acute prefrontal cortex transcranial magnetic stimulation in healthy volunteers: no effects on brain-derived neurotrophic factor (BDNF) concentrations in serum. J Affect Disord 107:255-258.

Lu LX, Yon JH, Carter LB, Jevtovic-Todorovic V (2006) General anesthesia activates BDNF-dependent neuroapoptosis in the developing rat brain. Apoptosis 11:1603-1615.

Malenka RC, Bear MF (2004) LTP and LTD: an embarrassment of riches. Neuron 44:5-21.
Martinowich K, Manji H, Lu B (2007) New insights into BDNF function in depression and anxiety. Nat Neurosci 10:1089-1093.

Müller MB, Toschi N, Kresse AE, Post A, Keck ME (2000) Long-term repetitive transcranial magnetic stimulation increases the expression of brainderived neurotrophic factor and cholecystokinin mRNA, but not neuropeptide tyrosine mRNA in specific areas of rat brain. Neuropsychopharmacology 23:205-215.

Nitsche MA, Roth A, Kuo MF, Fischer AK, Liebetanz D, Lang N, Tergau F, Paulus W (2007) Timing-dependent modulation of associative plasticity by general network excitability in the human motor cortex. J Neurosci 27:3807-3812.

Ouyang W, Hemmings HC Jr (2005) Depression by isoflurane of the action potential and underlying voltage-gated ion currents in isolated rat neurohypophysial nerve terminals. J Pharmacol Exp Ther 312:801-808.

Pell GS, Roth Y, Zangen A (2011) Modulation of cortical excitability induced by repetitive transcranial magnetic stimulation: influence of timing and geometrical parameters and underlying mechanisms. Prog Neurobiol 93:59-98.

Roche KW, O’Brien RJ, Mammen AL, Bernhardt J, Huganir RL (1996) Characterization of multiple phosphorylation sites on the AMPA receptor GluR1 subunit. Neuron 16:1179-1188.

Rossi S, Hallett M, Rossini PM, Pascual-Leone A (2009) Safety, ethical considerations, and application guidelines for the use of transcranial magnetic stimulation in clinical practice and research. Clin Neurophysiol 120:2008-2039.

Siebner HR (2010) A primer on priming the human motor cortex. Clin Neurophysiol 121:461-463.

Siebner HR, Hartwigsen G, Kassuba T, Rothwell JC (2009) How does transcranial magnetic stimulation modify neuronal activity in the brain? Implications for studies of cognition. Cortex 45:1035-1042.

Stehberg J, Levy D, Zangen A (2009) Impairment of aversive memory reconsolidation by localized intracranial electrical stimulation. Eur J Neurosci 29:964-969.

Taliaz D, Stall N, Dar DE, Zangen A (2010) Knockdown of brain-derived neurotrophic factor in specific brain sites precipitates behaviors associated with depression and reduces neurogenesis. Mol Psychiatry 15:80-92.

Thickbroom GW (2007) Transcranial magnetic stimulation and synaptic plasticity: experimental framework and human models. Exp Brain Res 180:583-593.

Toth E, Gersner R, Wilf-Yarkoni A, Raizel H, Dar DE, Richter-Levin G, Levit O, Zangen A (2008) Age-dependent effects of chronic stress on brain plasticity and depressive behavior. J Neurochem 107:522-532.

Vertes RP (2006) Interactions among the medial prefrontal cortex, hippocampus and midline thalamus in emotional and cognitive processing in the rat. Neuroscience 142:1-20.

Yukimasa T, Yoshimura R, Tamagawa A, Uozumi T, Shinkai K, Ueda N, Tsuji S, Nakamura J (2006) High-frequency repetitive transcranial magnetic stimulation improves refractory depression by influencing catecholamine and brain-derived neurotrophic factors. Pharmacopsychiatry 39:52-59.

Zanardini R, Gazzoli A, Ventriglia M, Perez J, Bignotti S, Rossini PM, Gennarelli M, Bocchio-Chiavetto L (2006) Effect of repetitive transcranial magnetic stimulation on serum brain derived neurotrophic factor in drug resistant depressed patients. J Affect Disord 91:83-86.

Zhang X, Mei Y, Liu C, Yu S (2007) Effect of transcranial magnetic stimulation on the expression of c-Fos and brain-derived neurotrophic factor of the cerebral cortex in rats with cerebral infarct. J Huazhong Univ Sci Technolog Med Sci 27:415-418. 